\title{
Influence of temperature on the refractive index sensitivities of fiber Bragg gratings refractometers
}

\author{
Diego Fernandes, Rosangela Winter, Jean Carlos C. da Silva and Ricardo C. Kamikawachi \\ Graduate Program in Electrical and Computer Engineering (CPGEI), Federal University of Technology - \\ Paraná, Curitiba, Brazil-Postal Code 80230-901.canute@utfpr.edu.br \\ Andreia G. Macedo \\ Graduate Program on Physics and Astronomy (PPGFA), Federal University of Technology - Paraná, Curitiba, \\ Brazil-Postal Code 80230-901
}

\begin{abstract}
Effect of cross-sensitivity between temperature and refractive index (RI) was studied using an optical sensor based on Bragg grating inscribed in an etched multi-mode fiber (MMF). RI sensitivities of different modes were used to obtain a linear response and temperature dependence in water:glycerin solutions. Results pointed out that the absolute average RI sensibility reduces from $0.97 \pm 0.05 \mathrm{~nm} / \mathrm{RIU}$ to $0.76 \pm 0.07 \mathrm{~nm} / \mathrm{RIU}$ while the temperature increases from $15^{\circ} \mathrm{C}$ up to $35^{\circ} \mathrm{C}$, respectively. Effect of temperature on the RI sensitivity is related with the increasing difference between the fiber and surrounding RI, which results in lower evanescent field and, consequently, reduced surrounding RI sensitivity. Therefore, in order to improve the precision during the RI measures, the effect of temperature must be considered in the calibration curves, principally in MMF fiber grating sensors that show low RI sensitivity values.
\end{abstract}

Index Terms - fiber Bragg gratings, refractive index sensitivity, temperature sensitivity, thermo-optic effect.

\section{INTRODUCTION}

Functional optical sensors for surrounding refractive index (RI) have been applied in quality control of industrialized food, chemicals or biochemicals. Moreover, devices based on optical fibers exhibit some advantages such as reduced size and weight, allow remote monitoring with high multiplexing capacity, electrical passivity and electromagnetic immunity [1-8]. However, due the thermo-optic effect, changes of temperature also induces RI variations at the fiber and/or at the response to the surrounding RI and consequently, sensors based on optical fibers present a cross sensibility that may afford imprecise responses.

Several temperature and RI optical sensors are reported at the literature, where the cross-sensibility effect has been suppressed by different devices geometries [1-4] or signal processing [5]. For instance, Z. Li et al. [1] proposed an intensity-modulated RI sensors based on in-fiber Michelson interferometer by splicing a section of thin core fiber between two standard single mode fiber (SMF) that are insensitive to temperature and thus, using this setup, the cross-sensitivity between temperature 
and RI was removed. These sensors displayed sensitivity of 208.24 and $125.44 \mathrm{~dB} / \mathrm{RIU}$ at the RI of 1.440 and 1.500, respectively, at temperature range between $25{ }^{\circ} \mathrm{C}$ and $100{ }^{\circ} \mathrm{C}$. Moreover, C. Gouveiaet.al [2] reported sensors based on a white light Mach-Zehnder configuration, with serrodyne phase modulation used to interrogate two similar non-adiabatic tapered optical fiber sensors in a differential scheme. In this situation, the system was able to measure the RI independent of temperature and they were able to detect a $\Delta \mathrm{RI} \approx 1.46 \times 10^{-6}$. The authors emphasized that the sensor arrangement still sensitive to the changes induced in the RI of the solution by the temperature, or equivalent, variations of RI derived from the thermo-optic coefficient at the solution were measured, while the effects of the silica thermo-optic coefficient in the fiber sensor were canceled out. A hybrid optical fiber structure for RI measurement was also reported [3], in this case the sensor was composed of single-multimode-single mode fibers (SMS) and a long period fiber grating (LPFG), as sensing mechanism the SMS structure was insensitive towards changes of RI, but sensitive to temperature, while the LPFG was sensitive to both parameters.

Different approaches to measure temperature and environmental RI were also reported at the literature [6-10]. Mostly, the cross-sensibility was studied considering the fiber Bragg grating (FBG) response as a combination of thermal and RI sensibility, but few works evaluate how the temperature variation affects the RI sensibility. Recently, H. Z. Yang et al. [5] showed that for accurate simultaneous measurement, the element of cross sensitivity between temperature and RI must be included in the measurement using $3 \times 3$ characteristic matrix, but they have not studied in detail how temperature affects RI sensitivity. Silica has positive thermo-optic coefficient and thus, a temperature increase induces a decrease in the evanescent field, reducing the Bragg grating sensibility to the changes in environmental RI.

Herein, influence of temperature on the RI sensitivity of a fiber Bragg grating inscribed in an etched MMF fiber was evaluated. RI sensitivities of different modes were used to obtain a linear response and a relation between this parameter and temperature. Results pointed out that the temperature increases induce a slight reduction of RI sensitivity.

\section{METHODOLOGY}

Solutions of water:glycerin were used to characterize the RI sensitivity, eight samples were prepared with glycerin concentration changing from 0 up to $80 \%(\mathrm{v} / \mathrm{v})$. RI values were measured using an ABBE refractometer (Biobrix - 2WAJ) with RI resolution of $0.5 \times 10^{-4}$. Additionally, in order to quantify the RI changes as a function of the temperature in the water:glycerin solutions, a thermostatic bath (Lauda ECO RE 415G) was coupled to the ABBE refractometer.

Sensor preparation: Bragg grating was recorded in a MMFfiber (Furukawa) having core diameter of $50 \mu \mathrm{m}$. The MMF was chosen because allows to obtain the RI sensitivity with a reduction of the fiber diameter lower than that required in a single mode fiber (SMF). Reduction of the fiber diameter was 
achieved using the following procedure: a surface corrosion was performed using an aqueous hydrochloric acid solution (HF 40\%), followed by a neutralization procedure using aqueous sodium hydroxide solution $(\mathrm{NaOH} 2 \mathrm{Mol} / \mathrm{L})$. The rate of corrosion was monitored in situ by the shift of the wavelength peak at the reflected signal. The FBG reflected spectrum is presented at Figure 1, this spectrum has three peaks, denoted as 1,2 and 3 modes, being the higher intensity peak associated with the fundamental mode [11]. A reproducible relation between the peak shift and the fiber diameter was found. Finally, the fiber diameter was measured using an optical microscope (OLYMPUS $\mathrm{BX} 51 \mathrm{RF})$, displaying that the final fiber diameter was $17 \mu \mathrm{m}$.

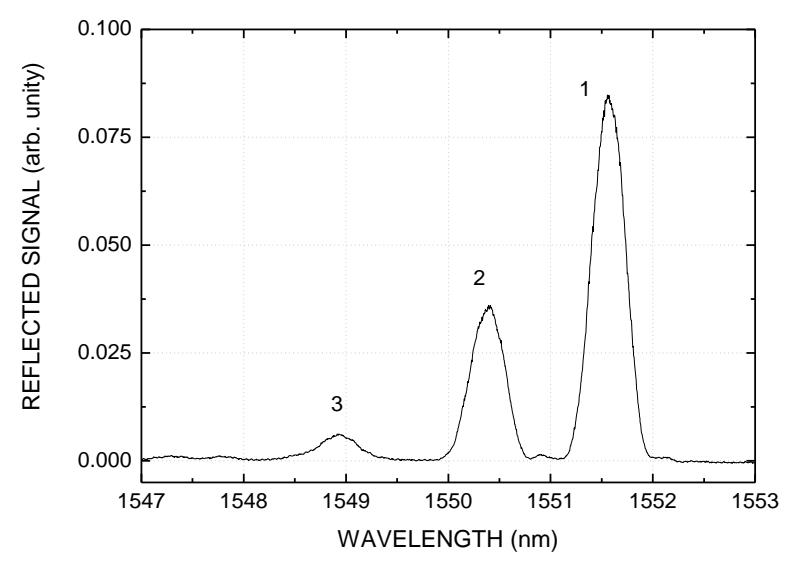

Fig. 1. Reflected spectrum acquired from a Bragg grating recorded on a MMF, after the corrosion procedure.

The sensor response was characterized using a SM125 interrogator (MicroOptics) with resolution of $\pm 1 \mathrm{pm}$. For these measurements, water:glycerin solutions were harvested in several glass tubes that allow the simultaneous insertion of a thermometer and the fiber sensor. This set-up was immersed in a thermostatic bath (Lauda) with controlled temperature. After each measure, the thermometer and the fiber sensor were washed with water and isopropyl alcohol (iPrOH). In order to confirm that the residuals were effectively removed, a reflection spectrum was acquired from the fiber sensor immersed in water and compared with a reference spectrum. The FBG RI response was monitored at temperatures of $15,20,25,30$ e $35^{\circ} \mathrm{C}$. RI was monitored at temperatures of $15,20,25,30$ e $35^{\circ} \mathrm{C}$. To ensure reproducibility, each sample was analyzed 5 times upon similar conditions.

\section{RESULTS AND DISCUSSION}

Figure 2 shows the RI variation of water-glycerin solutions as a function of the temperature, these results were acquired using the $\mathrm{ABBE}$ refractometer and thus, corresponds to a suitable RI dependence with temperature. The RI versus temperature curve presents a nonlinear behavior and a proper calibration curve was obtained by fitting a second order function over the experimental data. As can be observed, the RI values increase with glycerin content and decrease with temperature. 
In order to verify the effects of the temperature in the RI sensitivity the modes 1 and 2 were monitored. The peak positions of modes, as a function of RI and temperatures are showed at Figure 3. The changes in the RI values due to temperature increase were corrected using the calibration curves from figure 2.

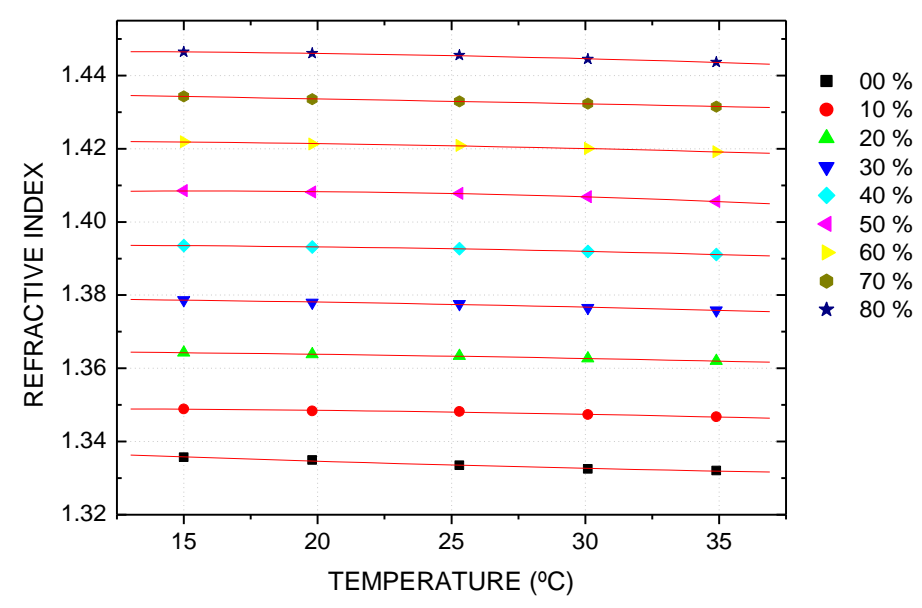

Fig. 2. RI versus temperature acquired from water:glycerin solutions using an ABBE refractometer coupled to a thermostatic bath (symbols are experimental data and the solid lines are fitting of a second order function).

Wavelength shifts of mode 2, due to the RI increase at different temperatures, are presented at Figure 4. In this figure, solid lines depict the best fittings to the empirical equation (1):

$$
\lambda=a_{1}-\frac{a_{2}}{\left(a_{3}-n\right)}
$$

Where $n$ is the surrounding (water-glycerin solution) RI and $a_{1}=-(0.31 \pm 0.12) \mathrm{nm}, a_{2}=-(0.089$ $\pm 0.054)$ and $a_{3}=(1.621 \pm 0.069)$ are the fitting parameters at $15^{\circ} \mathrm{C}$. These curves showed that the wavelength shifts decrease with temperature increasing. Error bars are combined standard uncertainties and were calculated according to standard uncertainties, inherent to the experimental standard deviation of the mean of FBG wavelength, measured 5 times under repeatability conditions, as well as the OSA resolution uncertainty, the Gaussian fitting uncertainty used to determine the FBG wavelength and the limit error inherent to the RI measurements. 


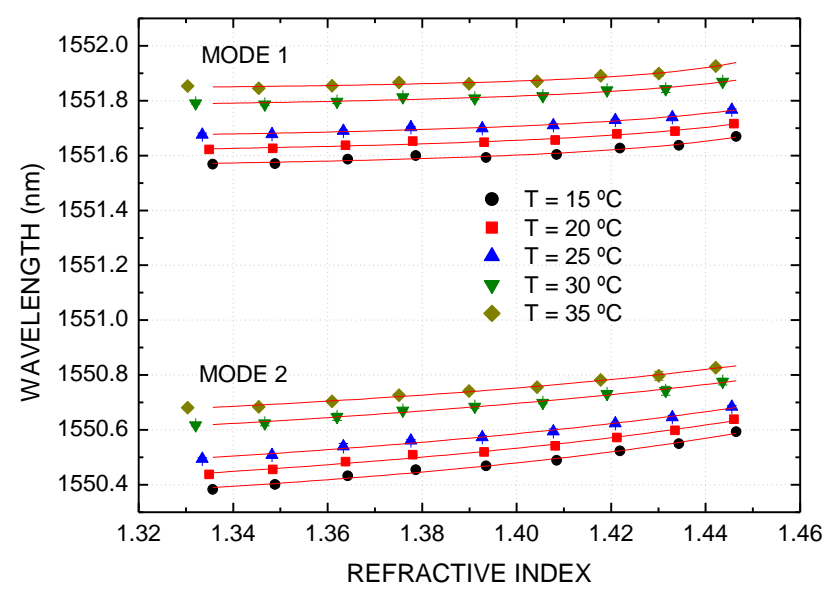

Fig. 3. Wavelength positions of modes 1 and 2, as a function of RI and temperature.

Figure 5 displays the sensitivity of mode 2 as a function of the RI and the temperature. These sensitivity curves are obtained from the differentiation of empirical fitting. Comparing these five sensitivity curves, acquired at temperature range between $15{ }^{\circ} \mathrm{C}$ and $35{ }^{\circ} \mathrm{C}$, it is clear that the sensitivity of mode 2 falls to lower values mainly at the higher temperature range.

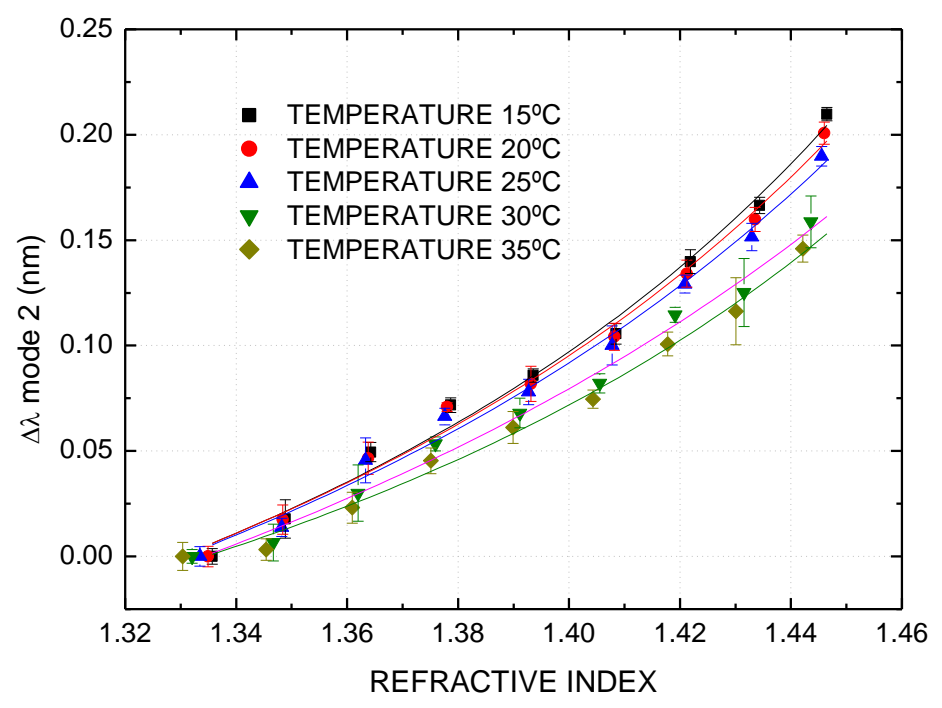

Fig. 4.Wavelength shifts of mode 2 against RI and temperature.

Sensor response to the changes of RI can also be obtained from the difference between the peak positions of mode 2 and mode $1\left(\lambda_{2}-\lambda_{1}\right)$. The evolution of this difference, against RI and temperature, is displayed at Figure 6, in this case a linear fitting was applied and resulted in correlation coefficients between -0.99713 and -0.99895 to $15^{\circ} \mathrm{C}$ and $35^{\circ} \mathrm{C}$, respectively. 


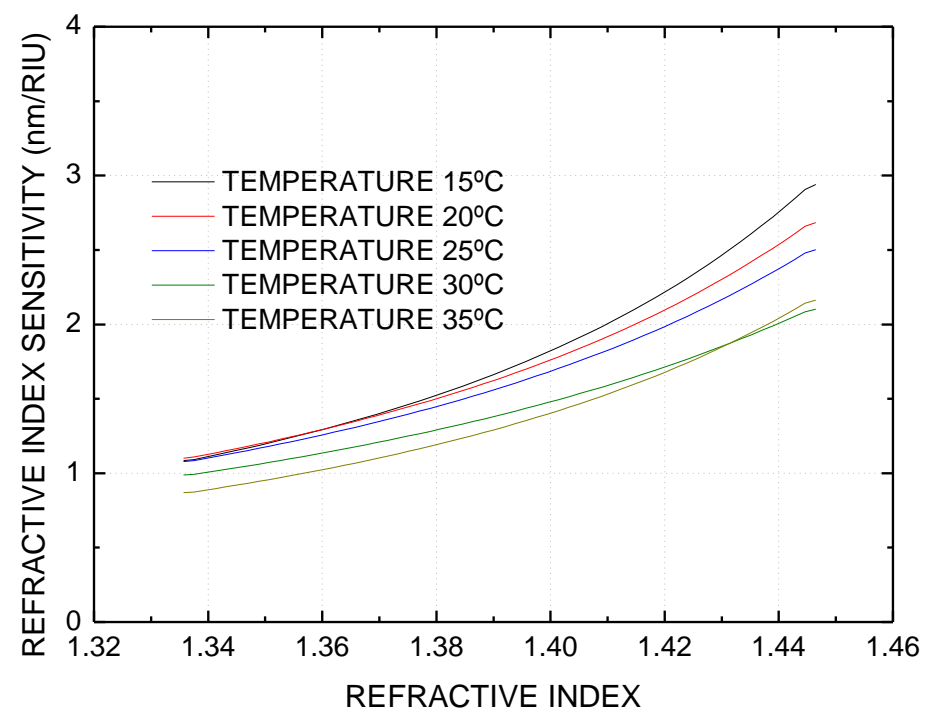

Fig. 5.RI sensitivity of mode 2 as a function of the temperature.

Figure 7 presents the changes of average RI sensitivity as a function of the temperature. The absolute average RI sensitivity reduces from $0.97 \pm 0.05 \mathrm{~nm} / \mathrm{RIU}$ to $0.76 \pm 0.07 \mathrm{~nm} / \mathrm{RIU}$ when the temperature increases from $15^{\circ} \mathrm{C}$ up to $35^{\circ} \mathrm{C}$, respectively, with a reduction of $0.2 \mathrm{~nm} / \mathrm{RIU}$ and a rate of $0.010 \pm 0.004 \mathrm{~nm} /\left(\mathrm{RIU}^{\circ} \mathrm{C}\right)$. The decrease in the RI sensitivity with the temperature increase can be explained by the weakening of the evanescent field. The increase in the temperature causes an increase in the fiber RI and, on the other hand, a decrease in the surrounding RI. As result, the evanescent wave becomes more confined reducing the RI sensitivity.

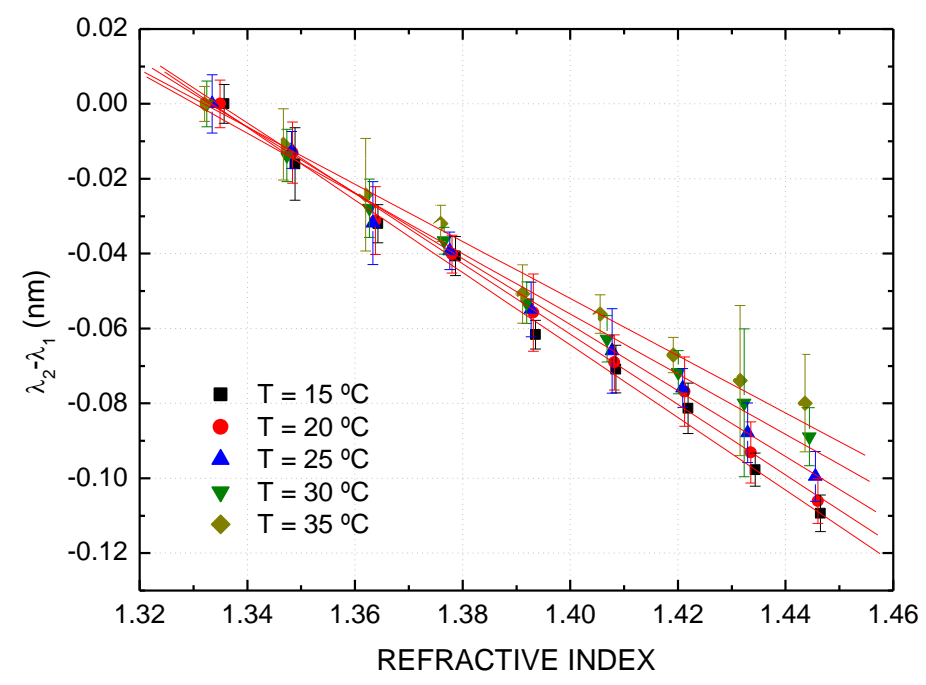

Fig. 6.Difference between the peak positions of mode 2 and mode 1 against RI and temperature.

Due the fact of this fiber Bragg grating MMF has low RI sensitivity; the effect of temperature on the RI sensitivity is not marginal and thus, must be considered in the calibration curves in order to improve the precision during the RI measures. 


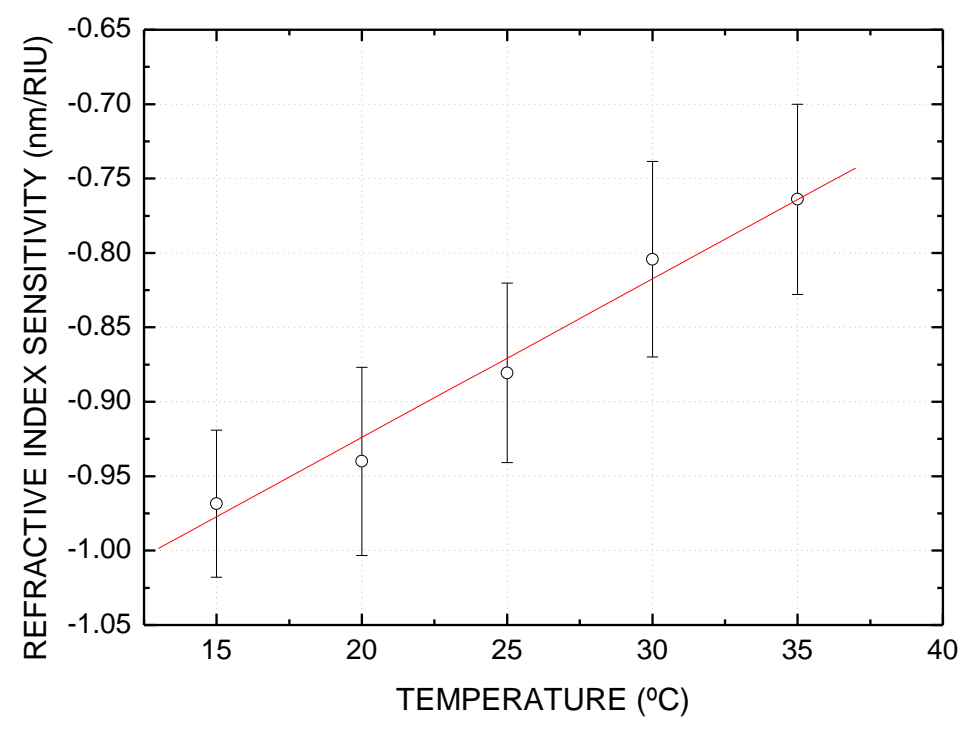

Fig. 7.Average FBG RI sensitivity against temperature.

\section{CONCLUSION}

It was verified that the increasing temperature induces a reduction of RI sensitivity of Bragg grating MMF with reduced diameter. This reduction was mainly attributed to a decrease in the evanescent field, resulting from the increased difference between the fiber and surrounding RI. Considering the thermo-optic coefficient for silica of around $8 \times 10^{-6}{ }^{\circ} \mathrm{C}^{-1}$, it is expected a low reduction of RI sensibility with the increase in the temperature, and due this, in typical applications were the RI sensitivity is at order of $10^{2} \mathrm{~nm} / \mathrm{RIU}$ this effect can be overlooked. However, if the sensor has low RI sensibility and displays other interesting features such as simultaneous measures, or if the application requires large temperature variations, this effect must be considered in the calibration curves. Monitoring the difference between the wavelength peaks position allow obtain a linear relation for the RI sensitivity as a function of the temperature resulting in a direct analysis of the cross sensitivity.

\section{ACKNOWLEDGMENT}

THE AUTHORS ACKNOWLEDGE FINEP, CAPES, SETI, CNPQ AND FUNDAÇÃOARAUCÁRIA FOR SCHOLARSHIPS AND FUNDING. THE USED FBG WERE RECORDED AT THE PHOTONICS LABORATORY OF UTFPR-CURITIBA, ASSOCIATED LABORATORY OF INCT FOTONICOM.

\section{REFERENCES}

[1] Zhengyong Li, Yiping Wang, Changrui Liao, Shen Liu, Jiangtao Zhou, Xiaoyong Zhong, Yingjie Liu, Kaiming Yang, Qiao Wang, Guolu Yin, "Temperature-insensitive refractive index sensor based on in-fiber Michelson interferometer", Sensors and Actuators B: Chemical, vol. 199 (2014) 31-35.

[2] Carlos Gouveia, Mohammad Zibaii, Hamid Latifi, Manuel J. B. Marques, J.M. Baptista, Pedro A.S. Jorge, "High resolution temperature independent refractive index measurement using differential white light interferometry", Sensors and Actuators B: Chemical, vol. 188, (2013) 1212-1217. 
[3] Jie Huang, Xinwei Lan, Amardeep Kaur, Hanzheng Wang, Lei Yuan, Hai Xiao, "Temperature compensated refractometer based on a cascaded SMS/LPFG fiber structure”, Sensors and Actuators B: Chemical, vol. 198 (2014) 384-387.

[4] Y. Wang, D.N. Wang, C.R. Liao, T. Hu, J. Guo, H. Wei, "Temperature-insensitive refractive index sensing by use of micro Fabry-Perot cavity based on simplified hollow-core photonic crystal fiber", Optics Letters, vol. 38, (2013) 269271.

[5] Hang Zhou Yang, Muhammad Mahmood Alib, Md Rajibul Islamb, Kok Sing Limb, Dinusha Serandi Gunawardena, Harith Ahmad, "Cladless few mode fiber grating sensor for simultaneous refractive index and temperature measurement", Sensors and Actuators A: Physical, vol 228 (2015) 62-68.

[6] A. Iadicicco, S. Campopiano, A. Cutolo, M. Giordano, A. Cusano, "Non uniform thinned fiber Bragg gratings for simultaneous refractive index and temperature measurements", IEEE Photonics Technology Letters, vol. 17 (2005) $1495-1497$.

[7] Xuan Guo, Weihong Bi, Lingxiao Wang, Feng Liu, "Simultaneous measurement of refractive index and temperature using dual-period grapefruit microstructured fiber grating”, Optik, vol. 124, (2013), pp. 3371-3374.

[8] Huihao Wang, Hongyun Meng, Rui Xiong, Qinghao Wang, Ben Huang, Xing Zhang, Wei Yu, Chunhua Tan, Xuguang Huang, "Simultaneous measurement of refractive index and temperature based on asymmetric structures modal interference", Optics Communications, vol. 364, (2016), pp. 191-194.

[9] Qiqi Yao, Hongyun Meng, Wei Wang, Hongchao Xue, RuiXiong, Ben Huang, Chunhua Tan, Xuguang Huang, "Simultaneous measurement of refractive index and temperature based on a core-offset Mach-Zehnder interferometer combined with a fiber Bragg grating", Sensors and Actuators A: Physical, vol. 209, (2014), pp. 73-77.

[10] P. Lu, L. Men, K. Sooley, Q. Chen, "Tapered fiber Mach-Zehnder interferometer for simultaneous measurement of refractive index and temperature, Applied Physics Letters, vol. 94 (2009) 1311101-1311103.

[11] Toru Mizunami, Tzvetanka V. Djambova, Tsutomu Niiho, Sanjay, "Bragg Gratings in Multimode and Few-Mode optical Fibers," Journal of Lightwave Technology vol. 18, (2000), pp. 230-235. 